\title{
Ultrafast Reorientation of Dangling OH Groups at the Air-Water Interface Using Femtosecond Vibrational Spectroscopy
}

\author{
Cho-Shuen Hsieh, ${ }^{1}$ R. Kramer Campen, ${ }^{1}$ Ana Celia Vila Verde, ${ }^{2, *}$ Peter Bolhuis, ${ }^{2}$ \\ Han-Kwang Nienhuys, ${ }^{1}$ and Mischa Bonn ${ }^{1,3, \dagger}$ \\ ${ }^{1}$ FOM Institute AMOLF, Science Park 104, 1098 XG Amsterdam, The Netherlands \\ ${ }^{2}$ Van 't Hoff Institute for Molecular Science, University of Amsterdam, Science Park 904, 1098 XH Amsterdam, The Netherlands \\ ${ }^{3}$ Max Planck Institute for Polymer Research, Ackermannweg 10, 55128 Mainz, Germany.
}

(Received 16 June 2011; published 8 September 2011)

\begin{abstract}
We report the real-time measurement of the ultrafast reorientational motion of water molecules at the water-air interface, using femtosecond time- and polarization-resolved vibrational sum-frequency spectroscopy. Vibrational excitation of dangling $\mathrm{OH}$ bonds along a specific polarization axis induces a transient anisotropy that decays due to the reorientation of vibrationally excited $\mathrm{OH}$ groups. The reorientation of interfacial water is shown to occur on subpicosecond time scales, several times faster than in the bulk, which can be attributed to the lower degree of hydrogen bond coordination at the interface. Molecular dynamics simulations of interfacial water dynamics are in quantitative agreement with experimental observations and show that, unlike in bulk, the interfacial reorientation occurs in a largely diffusive manner.
\end{abstract}

PACS numbers: 82.65.+r, 33.70.Ca, 36.20.Ng, 82.80.Gk

A molecular level understanding of how water meets surfaces is important for many topics in electrochemistry, atmospheric chemistry, and membrane biophysics. These interfaces require the termination of the bulk water hydrogen-bonded network and thus have unique water structure and properties, e.g., water's high surface tension. While always different from bulk, the specific properties of interfacial water depend on the adjoining phase. Of particular interest, both because of its ubiquity and its relationship to the solvation of hydrophobic phases more generally, are the properties of water at the air-water interface.

The structure of water at the water-air interface has been studied extensively both experimentally and theoretically [1-3]. These studies have shown that the interfacial width is $\approx 1 \mathrm{~nm}$ [3], and that $20 \%-25 \%$ of the surface is covered with non-hydrogen-bonded, free $\mathrm{OH}$ groups, sticking into the vapor phase [1,2].

However, in analogy to the bulk, interfacial molecules are expected to be structurally dynamic over ps time scales. Insight into these dynamics is essential for a complete understanding of interfacial water and interfacial hydration processes. Because the free $\mathrm{OH}$ is the least coordinated of aqueous species and is a general feature of water near hydrophobic surfaces [4], it seems likely that the dynamics of this water species play an important role in determining the air-water interface reactivity and in the molecular mechanisms of hydrophobic solvation [5]. While water near small hydrophobic groups has been shown to slow down relative to bulk water [6] in both experiment and simulation, the dynamics of water near extended surfaces are substantially less studied. In a recent simulation study of an idealized extended hydrophobic surface a population of water molecules was identified with one $\mathrm{OH}$ group pointing away from bulk liquid whose dynamics were sped up relative to bulk [7].

Here, we present the first experimental measurement of water structural dynamics at an extended interface. We find that rotation of interfacial free $\mathrm{OH}$ is accelerated $3 \times$ relative to bulk water. The experimental results are complemented by molecular dynamics simulations that are in quantitative agreement and shed additional light on the mechanism of interfacial reorientation.

We probe the rotational motion of water at the waterair interface using femtosecond time- and polarizationresolved vibrational sum-frequency (VSF) spectroscopy, an extension of time-resolved VSF spectroscopy [8]. In a conventional VSF experiment, infrared and visible laser pulses are overlapped in space and time on an aqueous surface, and the sum frequency of the two laser fields, generated selectively at the surface, detected. If the infrared light is resonant with the $\mathrm{OH}$ stretch vibration of surface water, this process is resonantly enhanced and the vibrational spectrum of the outermost few layers of water molecules recorded (i.e., VSF spectroscopy). A time-averaged VSF spectrum of the $\mathrm{OH}$ stretch frequency window at the air-water interface is shown in Fig. 1(a). As first shown by Du and Shen, this spectrum contains a broad feature at lower frequencies that is due to interfacial $\mathrm{OH}$ groups that are hydrogen-bonded, and a narrow peak near $3700 \mathrm{~cm}^{-1}$ that is the response of $\mathrm{OH}$ groups that are non-hydrogen-bonded (i.e., free $\mathrm{OH}$ ) and point towards the vapor $[1,9]$. In this study we employ a time- and polarization-resolved IR pump/VSF probe scheme in which the frequency of the pump and probe IR pulses is centered on the free $\mathrm{OH}$ : we vibrationally excite a orientational subset of free $\mathrm{OH}$ groups 

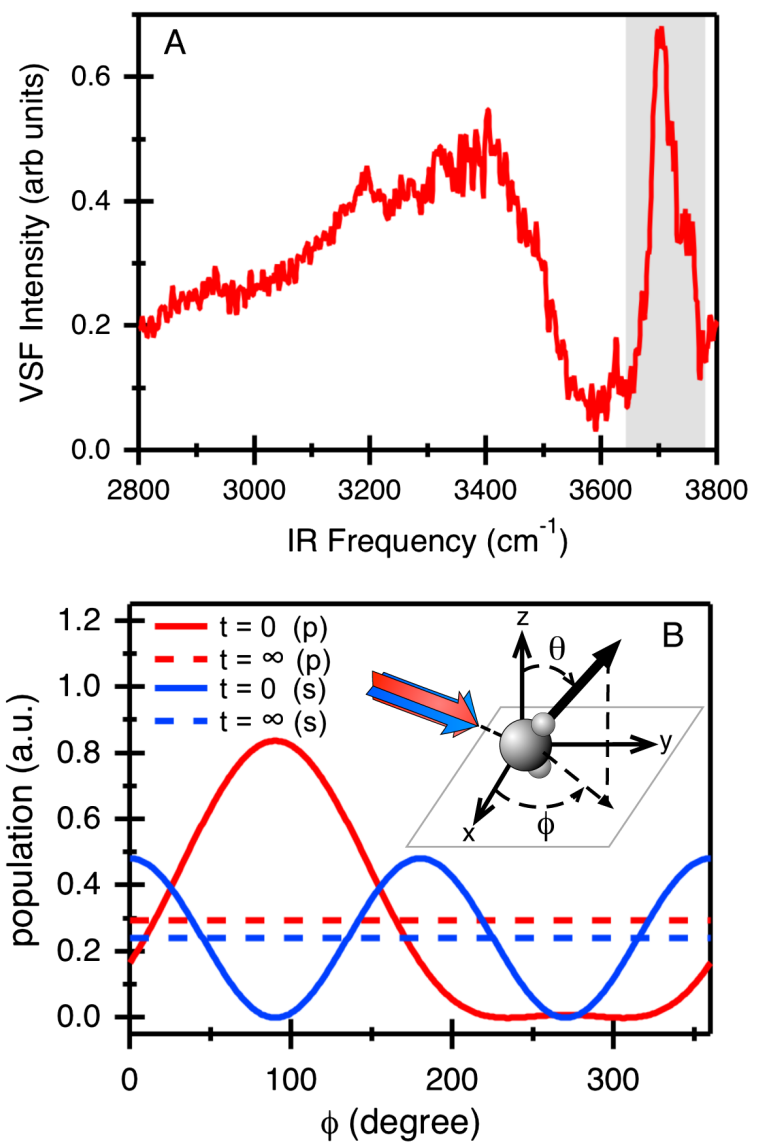

FIG. 1 (color online). (A) Time-averaged VSF spectrum of the air- $\mathrm{H}_{2} \mathrm{O}$ interface. In our IR pump/VSF probe experiment, only the free $\mathrm{OH}$ (grey rectangle) is pumped and probed. (B) Distribution of excited $\mathrm{OH}$ groups as a function of $\phi$ for $s$ and $p$ polarized IR pump at pump/probe overlap $(t=0)$ and at long pump/probe delays for $\theta_{m}=59^{\circ}$ and $\Delta \theta=26^{\circ}$ and the experimental IR pump angle. Inset: Experimental geometry. The $p$-polarized pump has its $E$ field in the $y z$ plane.

and track their vibrational and reorientational relaxation. Previous theoretical efforts by us and others have shown that such experiments provide insight into interfacial reorientational motion [10,11]. The IR pump/VSF probe setup is described elsewhere and in the Supplemental Material $[12,20]$.

A representative IR pump/VSF probe result is shown in Fig. 2. In this experiment, VSF spectra are recorded for $s, s$, and $p$ polarizations for the VSF, visible, and IR probe, respectively, at different delay times between pump and probe. Polarizations are defined relative to the $y z$ plane (i.e., the plane of incidence). The differential IR pump/VSF probe signal is computed as the ratio between the integrated intensities [in grey area in Fig. 1(a)] with and without the pump. The two traces are recorded in one experimental run. For both pump polarizations excitation of $\mathrm{OH}$ groups from the $\nu=0$ vibrational ground state to the $\nu=1$ first vibrationally excited state leads to a transient decrease in the VSF signal (the VSF signal is shifted

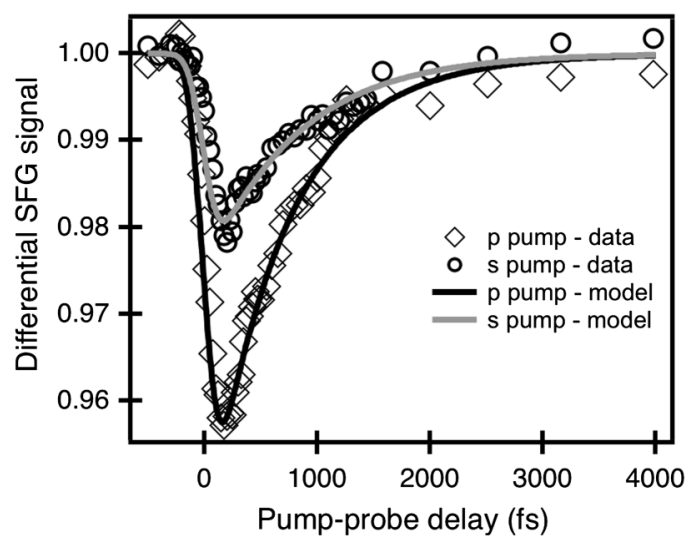

FIG. 2. IR pump/VSF probe data for free $\mathrm{OH}$ at air-water interface with $s$ - and $p$-polarized pump. The black and grey lines are generated using the numerical model of Nienhuys and Bonn with the parameters $D_{\phi}=0.32 \frac{\mathrm{rad}^{2}}{\mathrm{ps}}, D_{\theta}=0.36 \frac{\mathrm{rad}^{2}}{\mathrm{ps}}$, $\tau_{\nu}=800 \mathrm{fs}$, average angle $\theta_{m}=59^{\circ}$, and FWHM of angular distribution $\Delta \theta=26^{\circ}$.

out of our frequency window due to the anharmonicity of the vibration). Two important observations can be made from the raw data: (i) at zero delay time, significant anisotropy exists between $s$ - and $p$-polarized pump transients and (ii) the recovery of the differential VSF signals is significantly faster for the $p$-polarized pump trace (640 $\pm 40 \mathrm{fs}$ ) than the $s$-polarized $(870 \pm 100 \mathrm{fs}$; uncertainty is $2 \sigma$, see Supplemental Material [20]).

Regarding (i), the initial anisotropy can be understood by noting that when the IR pump and IR-VSF probe are polarized parallel (both $p$ polarized), those $\mathrm{OH}$ groups that are most efficiently pumped (pump efficiency $\left.\propto\left|\mu_{\mathrm{OH}} \cdot E_{\text {pump }}\right|^{2}\right)$ are also most efficiently detected; when the pump and the IR portion of the VSF probe are orthogonally polarized, the differential signal is smaller. More specifically, Fig. 1(b) shows that for parameters relevant to our experiment (see discussion below) - angle of incidence of IR pump $50^{\circ}$, and average angle of $\mathrm{OH}$ groups with surface normal $\theta_{m}=59^{\circ}$ and full width at half maximum (FWHM) distribution $\Delta \theta=26^{\circ}$-at $t=0$ the excited state population with $p$-polarized IR pump is larger and exhibits an angular distribution dramatically different from that for the $s$-pumped case (the $p$-pumped distribution peaks at $\phi=90^{\circ}$ while the $s$-pumped peaks at $\phi=0^{\circ}$ and $\phi=180^{\circ}$ ). Note that while the quantitative details of excited population as a function of $\phi$ are a function of molecular orientation, the qualitative result, more population excited under the $p$ pump than the $s$ and a greater variability in the $p$ excited population with changing $\phi$, is not. These features will occur for any interfacial moiety that has a preferred distribution with respect to $\theta$ but not with respect to $\phi-$ e.g., any liquid-under excitation by an IR pump in reflection geometry. To actually calculate the VSF response at $t=0$ requires additionally accounting for the hyperpolarizability tensor, Fresnel factors, and angles 
of the probe beams. As shown in Fig. 2 and discussed below, such a calculation reproduces our data quite well and thus tightly constrains the possible average and distribution of $\theta$ for the free $\mathrm{OH}$ : the larger bleach seen in the $p$-compared to the $s$-pumped signals at time zero sensitively depends on free $\mathrm{OH}$ orientation.

Regarding (ii), the differences in rate of signal recovery as a function of pump polarization can be understood as follows. In principle both $p$ - and $s$-pumped traces are influenced by reorientational motion and vibrational relaxation (with time scale $\tau_{\nu}$ ). $\tau_{\nu}$ is expected to be independent of pump pulse polarization. This suggests that the differences in rate of signal recovery are a function of reorientation. Indeed, one would expect the $p$ channel to exhibit substantially more decay due to reorientation than the $s$ channel, in analogy to bulk anisotropy measurements: the $p$-polarized pump pulse preferentially excites those $\mathrm{OH}$ groups that are efficiently detected with the VSF probe pair that also contains a $p$-polarized IR-VSF probe pulse. As these $\mathrm{OH}$ groups reorient away from angles where they are efficiently detected, the signal will decay. In contrast, for the $s$-polarized pump, reorientation may cause motion towards orientations to which the $p$-polarized IR-VSF probe pulse is sensitive.

In principle, reorientational relaxation can also be due to rapid intermolecular excitation transfer. Such dipoledipole excitation transfer between differently oriented $\mathrm{OH}$ groups is known to dominate the polarization anisotropy measured, in polarization-resolved ultrafast transient IR absorption experiments for pure $\mathrm{H}_{2} \mathrm{O}[13,14]$. Estimates of its rate (assuming a Förster type dipole-dipole coupling mechanism), however, suggest that the characteristic time scale of excitation transfer for free $\mathrm{OH}$ at the air-water interface exceeds 20 ps: our observables are influenced only by reorientation and vibrational relaxation.

Prior theoretical analysis [10] suggests that our signal responds principally to reorientation in the plane of the interface (i.e., change in $\phi$ ), vibrational relaxation $\left(\tau_{\nu}\right)$, and average angle with respect to the surface normal $\left(\theta_{m}\right)$. Armed with this insight, we initially quantify reorientation rate of the free $\mathrm{OH}$ by assuming that the distribution of $\theta$ is a delta function and that there is no out-of-plane reorientation. The observed difference between the magnitude of the $s$ - and $p$-polarized differential VSF signals is particularly sensitive to the angle $\theta$; fits of our data with an assumed delta function distribution suggest $\theta=55^{\circ}$. If we further assume that the in-plane reorientational motion is diffusive and that vibrational relaxation occurs exponentially with a single time constant, fitting the data following the numerical proscription offered previously [10] suggests that the free $\mathrm{OH}$ in-plane angular diffusivity is $D_{\phi}=1.25 \pm 0.2 \frac{\mathrm{rad}^{2}}{\mathrm{ps}}$ and $\tau_{\nu}=850 \mathrm{fs}$. The diffusivity of $D_{\phi}=1.25 \frac{\mathrm{rad}^{2}}{\mathrm{ps}}$ implies the free $\mathrm{OH}$ reorients $>10 \times$ faster than that in the bulk (see [15] and below).
However, prior polarization-resolved static VSF studies and simulation studies suggest that $\theta$ assumes a distribution with a FWHM of at least $10^{\circ}$ [9]. Such a distribution suggests that any model of the spectroscopic response should include out-of-plane motion of the dangling OHs. In addition, recent studies of $\mathrm{OH}$ reorientation in bulk water have found that angular motion is nondiffusive $[16,17]$ over picosecond time scales. Because we are interested in free $\mathrm{OH}$ motion over the same time scales, our assumption of diffusive reorientation may rightly be questioned. To investigate both the effect of a finite angular distribution of $\mathrm{OH}$ groups and the potential nondiffusive nature of surface reorientation in more detail, we turn to classical all-atom simulation of the air-water interface using the extended simple point charge (SPC/E) water model (for details see Supplemental Material [20]). Within the simulation, histograms of $\theta$ for free $\mathrm{OH}$ groups at the interface show a fairly broad angular distribution centered near $\approx 55^{\circ}$ (see Supplemental Material [20]). This distribution implies that there is an effective potential that restrains the angular motion of the free $\mathrm{OH}$ along the surface normal and that physically valid modeling of our experimental results requires both out-of-plane and inplane motion.

But is the motion of surface water molecules diffusive? Regardless of mechanism, we expect that in-plane reorientation should be unhindered by a potential. As described by Einstein and Debye, the mean square angular displacement $\left(\left\langle\phi^{2}\right\rangle\right)$ should increase linearly with time for diffusive motion; a log-log plot of $\left(\left\langle\phi^{2}\right\rangle\right)$ vs time should produce a line with a slope of one. Figure 3 shows such a plot for both in-plane angular reorientation of free $\mathrm{OH}$ and reorientation of $\mathrm{OH}$ in bulk water (within the SPC/E model where reorientation in bulk is defined with respect to the angle $\omega$ of rotation from time $t=0$ to $t=\tau$ ). As illustrated, the in-plane angular motion of the free $\mathrm{OH}$, while not exactly

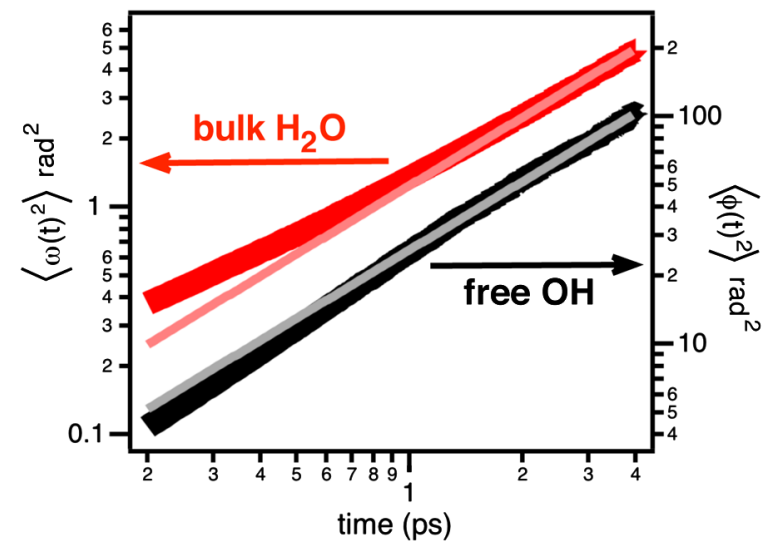

FIG. 3 (color online). Mean square angular displacement $\left\langle\phi^{2}\right\rangle$ of the free $\mathrm{OH}$ and of $\mathrm{OH}$ in bulk water $\left(\left\langle\omega^{2}\right\rangle\right)$. Thick lines are simulation results. Thin, lighter colored lines are fits to these results assuming a purely diffusive behavior. The diffusive approximation works well for the free $\mathrm{OH}$. 
diffusive, is far nearer to this behavior than that of $\mathrm{OH}$ in bulk. As discussed above, if out-of-plane motion is also diffusive it will be within a potential. In such systems mean square angular displacement approaches a constant value with time [18]. As is shown in the Supplemental Material [20], plots of $\left\langle\theta^{2}\right\rangle$ for the free $\mathrm{OH}$ show this behavior.

Motivated by the success of the SPC/E potential in reproducing bulk water dynamics [17], we also extracted the relevant values of the parameters for diffusive reorientation of interfacial water from the simulation. This can be accomplished using standard procedures for four parameters: the average out-of-plane orientation angle $\left(\theta_{m}\right)$, the FWHM of its distribution $(\Delta \theta)$, the in-plane diffusivity $\left(D_{\phi}\right)$, and the out-of-plane diffusivity $\left(D_{\theta}\right)$. The fifth-the vibrational relaxation time $\left(\tau_{\nu}\right)$-describes the disappearance of the excitation from the free $\mathrm{OH}$. This disappearance can be due to the redistribution of vibrational energy over the system's degrees of freedom (true vibrational relaxation), but may also be the result of the free $\mathrm{OH}$ becoming hydrogen-bonded after rotating into the liquid, or of (intramolecular) energy transfer into an $H$-bonded $\mathrm{OH}$ group. In both latter cases, the frequency of the excited vibration shifts out of our spectral window and does not affect the differential relaxation of the $p$ - and $s$ - pumped signals. The time scales of energy flow cannot be obtained from our classical simulations. If, however, vibrational relaxation is slow relative to rotation, estimates of $\tau_{\nu}$ based on the time the free $\mathrm{OH}$ remains without an $H$ bond in the simulations should describe our data well. Assuming $\tau_{\nu}$ describes the lifetime of the free $\mathrm{OH}$ due to rotation (see Supplemental Material for lifetime calculation [20]), the five parameters for our model as extracted from SPC/E simulations of free $\mathrm{OH}$ are $D_{\phi}=0.32 \frac{\mathrm{rad}^{2}}{\mathrm{ps}}, D_{\theta}=0.36 \frac{\mathrm{rad}^{2}}{\mathrm{ps}}$, $\tau_{\nu}=800 \mathrm{fs}, \theta_{m}=59^{\circ}$, and $\Delta \theta=26^{\circ}$ [19]. Armed with these parameters, a detailed description of how to calculate the signal has been offered previously [10]. Figure 2 shows the application of this model to the data. As is clear from inspection, the physical characteristics of the free $\mathrm{OH}$ from the simulation allow a quantitative description of the free $\mathrm{OH}$ dynamics with no adjustable parameters.

Direct comparison of our experimental data to polarization-resolved transient infrared absorbance of water in bulk is difficult as IR pump-VSF probe measurements in our experimental geometry sample a sum of correlation functions not equivalent to that measured in the former technique [11]. Similarly, comparison of our extracted $D_{\phi}$ and $D_{\theta}$ of the free $\mathrm{OH}$ to bulk is difficult because the motion of bulk water is known to be nondiffusive over our experimental time window [16,17]. Taking advantage of the ability of the SPC/E potential to simulate reorientational dynamics of bulk water in quantitative agreement with experiment, we can impose an arbitrary reference frame on bulk water and extract a $D_{\phi}$ and $D_{\theta}$ consistent with simulation that reproduces known experimental constraints (see Supplemental Material for details [20]). Such a calculation gives $D_{\phi, \text { bulk }}=D_{\theta, \text { bulk }}=$ $0.1 \frac{\mathrm{rad}^{2}}{\mathrm{ps}}$ : the free $\mathrm{OH}$ reorients $\approx 3 \times$ faster than does water in bulk. This speed up of water rotation at the air-water interface is in direct contrast to the slowdown of the rotation observed near small hydrophobic solutes and in qualitative agreement with a prior computational study of water near an idealized hydrophobic surface [7].

Prior work has shown that increasing free energy of wetting (for an increasingly hydrophobic surface) correlates with the creation of a population of increasingly dynamically labile interfacial water molecules. To this point only dynamical properties averaged over long times or many water molecules have been described: there has been no insight into how individual water molecules might contribute to this behavior. Here we show, for the first time experimentally, that these macroscopic time-averaged changes are accompanied by a change in molecular reorientation: the water-air interface is characterized by a population of dangling $\mathrm{OH}$ groups that, in contrast to water near both small hydrophobic and hydrophilic solutes, rotates dramatically faster than bulk.

This work is part of the research program of the Stichting Fundamenteel Onderzoek der Materie with financial support from the Nederlanse Organisatie voor Wetenschappelijk Onderzoek. We thank SARA Computing and Networking Services (www.sara.nl) for their support in using the Lisa Compute Cluster.

*Also at University of Minho, Physics Center, Campus de Gualtar, 4710-057 Braga, Portugal.

†bonn@amolf.nl

[1] Q. Du et al., Phys. Rev. Lett. 70, 2313 (1993).

[2] I. V. Stiopkin et al., Nature (London) 474, 192 (2011).

[3] G. Richmond, Annu. Rev. Phys. Chem. 52, 357 (2001); J. Penfold, Rep. Prog. Phys. 64, 777 (2001); T. D. Kühne et al., J. Phys. Chem. Lett. 2, 105 (2011).

[4] L. Scatena, M. Brown, and G. Richmond, Science 292, 908 (2001).

[5] D. Chandler, Nature (London) 437, 640 (2005); R. Godawat, S. N. Jamadagni, and S. Garde, Proc. Natl. Acad. Sci. U.S.A. 106, 15119 (2009).

[6] J. Qvist and B. Halle, J. Am. Chem. Soc. 130, 10345 (2008); D. Laage, G. Stirnemann, and J. T. Hynes, J. Phys. Chem. B 113, 2428 (2009); K.-J. Tielrooij et al., J. Am. Chem. Soc. 132, 15671 (2010).

[7] G. Stirnemann, P. J. Rossky, J. T. Hynes, and D. Laage, Faraday Discuss. 146, 263 (2010).

[8] J. McGuire and Y. R. Shen, Science 313, 1945 (2006); M. Smits et al., Phys. Rev. Lett. 98, 098302 (2007); A. Eftekhari-Bafrooei and E. Borguet, J. Am. Chem. Soc. 131, 12034 (2009); M. Bonn et al., ibid. 132, 14971 (2010).

[9] W. Gan et al., J. Chem. Phys. 124, 114705 (2006).

[10] H.-K. Nienhuys and M. Bonn, J. Phys. Chem. B 113, 7564 (2009). 
[11] Z. Gengeliczki, D.E. Rosenfeld, and M.D. Fayer, J. Chem. Phys. 132, 244703 (2010).

[12] A. Ghosh et al., Rev. Sci. Instrum. 79, 093907 (2008).

[13] S. Woutersen and H. Bakker, Nature (London) 402, 507 (1999).

[14] D. Kraemer et al., Proc. Natl. Acad. Sci. U.S.A. 105, 437 (2008).

[15] M. Mazza et al., Phys. Rev. Lett. 96, 057803 (2006).

[16] D. Laage and J. T. Hynes, Science 311, 832 (2006).

[17] D. Laage and J. T. Hynes, J. Phys. Chem. B 112, 14230 (2008).

[18] R. Metzler and J. Klafter, Phys. Rep. 339, 1 (2000).

[19] Our diffusion model assumes that motions in $\theta$ and $\phi$ are uncorrelated. While the quantitative ability to reproduce simulation (and experiment) with this model justifies this assumption, the small difference between $D_{\phi}$ and $D_{\theta}$ extracted from the simulation is also consistent with a scenario in which motions in these two coordinates are slightly correlated: possibly because the free $\mathrm{OH}$ reorients around the other, hydrogen-bonded $\mathrm{OH}$ on the molecule.

[20] See Supplemental Material at http://link.aps.org/ supplemental/10.1103/PhysRevLett.107.116102 for experimental and simulation details, fraction of free $\mathrm{OH}$ groups in the water slab, histograms of $\theta$ as a function of the lifetime of the free $\mathrm{OH}$, mean square $\theta$ displacement, average trajectory in $\theta$, dependence of chi-square on the in-plane and out-of-plane diffusion constants, discussion of model parameter uncertainty, and estimates of intermolecular energy transfer. 